\title{
LncRNA ZFAS1 serves as a prognostic biomarker to predict the survival of patients with ovarian cancer
}

\author{
SHUANG HAN ${ }^{1 *}$, DE-ZHAN $\mathrm{LI}^{2 *}$ and MEI-FANG XIAO ${ }^{3}$ \\ Departments of ${ }^{1}$ Gynaecology and ${ }^{2}$ Anesthesiology, Jingzhou Central Hospital, The Second Clinical Medical College, \\ Yangtze University, Jingzhou, Hubei 434020; ${ }^{3}$ Department of Clinical Laboratory, Center for Laboratory \\ Medicine, Hainan Women and Children's Medical Center, Haikou, Hainan 570206, P.R. China
}

Received August 21, 2018; Accepted May 2, 2019

DOI: $10.3892 /$ etm.2019.8135

\begin{abstract}
Ovarian cancer (OC) is one of the most fatal types of gynecological malignancy. Certain long non-coding RNAs (lncRNA) have been reported to have crucial roles in cancer progression. Zinc finger nuclear transcription factor, X-box binding 1-type containing 1 antisense RNA 1 (ZFAS1) is a novel regulator lncRNA in various cancer types. The expression pattern of most lncRNAs, including ZFAS1, in $\mathrm{OC}$ remains to be determined. In the present study, it was demonstrated that ZFAS1 was overexpressed in OC vs. normal cell lines. However, ZFAS1 was downregulated in OC compared with normal samples in the GEPIA dataset. Furthermore, OC samples of higher stages (stage III/IV) had higher levels of ZFAS1 compared with those in early-stage OC (stage I/II) samples. Of note, higher ZFAS1 expression was associated with shorter overall survival time and disease-free survival time of OC patients. Protein-protein interaction networks of proteins co-expressed with ZFAS1 in OC were constructed. Furthermore, Gene Ontology and Kyoto Encyclopedia of Genes and Genomes analysis of genes co-expressed with ZFAS1 indicated that ZFAS1 is associated with translation, mRNA splicing, cell-cell adhesion, DNA repair, protein sumoylation, positive regulation of GTPase activity and DNA replication. The present study may provide novel clues to validate ZFAS1 as a biomarker in $\mathrm{OC}$.
\end{abstract}

Correspondence to: Professor Mei-Fang Xiao, Department of Clinical Laboratory, Center for Laboratory Medicine, Hainan Women and Children's Medical Center, 15 Longkun South Road, Qiongshan, Haikou, Hainan 570206, P.R. China

E-mail: xiaomeifang2006@hotmail.com

*Contributed equally

Key words: zinc finger nuclear transcription factor, X-box binding 1-type containing 1 antisense RNA 1, protein-protein interaction, long non-coding RNA, ovarian cancer, biomarker

\section{Introduction}

Ovarian cancer (OC) is one of the most fatal types of gynecological malignancy. According to a recent survey, OC caused 14,240 deaths in 2017 worldwide (1). However, the mechanisms underlying OC progression have remained largely elusive. Long non-coding RNAs (lncRNA) are a novel class of RNA transcripts with a length of $>200$ nucleotides that lack any protein-coding capacity. Emerging studies have demonstrated that certain IncRNAs are dysregulated in tumor cells and have crucial roles in the progression of cancer, including prostate cancer, breast cancer, glioma and OC (2). Mechanistically, IncRNAs bind to RNAs, DNA and proteins to regulate multiple biological functions, including cell proliferation, apoptosis, epithelial-mesenchymal transition and cell migration. Several lncRNAs have been reported to be associated with OC progression. For instance, LINK-A was reported to promote $\mathrm{OC}$ cell migration and invasion by activating the transforming growth factor- $\beta$ pathway (3). Furthermore, Shu et al (4) indicated that lncRNA regulator of Akt signaling associated with hepatocellular carcinoma (HCC) and renal cell carcinoma promoted OC cell proliferation and invasion by association with $\mathrm{HuR}$ and the microRNA (miRNA/miR)-200 family. Therefore, exploring the expression pattern of IncRNAs in OC is useful for the identification of novel biomarkers for OC.

LncRNA zinc finger nuclear transcription factor, $X$-box binding 1-type containing 1 antisense RNA 1 (ZFAS1) has been reported to be upregulated in several types of cancer, including HCC, colorectal cancer, gastric cancer and glioma. In gastric cancer, ZFAS1 was demonstrated to be involved in regulating cancer progression through influencing $\mathrm{Wnt} / \beta$-catenin signaling and epigenetically repressing Kruppel-like factor 2 (KLF2) and NKD inhibitor of WNT signaling pathway 2 (NKD2) (5). In colorectal cancer, ZFAS1 promotes proliferation and metastasis via regulating zinc finger E-box binding homeobox 1 (ZEB1) expression and interacting with cyclin D kinase 1 (6). ZFAS1 was reported to promote OC cell malignancy by interacting with miR-150-5p (7). These reports suggested ZFAS1 may serve as a regulator in cancer progression. However, the expression pattern and functional roles of ZFAS1 in OC progression have remained to be elucidated. 
In the present study, the expression levels of ZFAS1 were compared between OC and normal samples and the association of ZFAS1 expression with clinicopathological features in OC was determined by analyzing publicly available datasets. The potential biological functions of ZFAS1 were analyzed by performing a Bioinformatics analysis. The present study may provide clues that validate ZFAS1 as a novel biomarker for OC.

\section{Materials and methods}

Dataset analysis. The raw data were obtained from The Cancer Genome Atlas (TCGA) data from Genomic Data Commons Data Portal (portal.gdc.cancer.gov) and the University of California Santa Cruz (UCSC) Xena project (http://xena.ucsc. edu). The data were analyzed using Gene Expression Profiling Interactive Analysis (GEPIA) (8), a newly developed interactive web server for analyzing RNA sequencing (RNA-seq) expression data. RNA-seq datasets used by GEPIA are based on the UCSC Xena project, using a standard processing pipeline. $\mathrm{P}<0.05$ was selected as the cutoff for identifying significantly differentially expressed genes.

Protein-protein interaction (PPI) network construction and analysis. In the present study, genes co-expressed with ZFAS1 were determined. The Starbase (9) and TargetScan $(10,11)$ databases were used to predict the miRNAs targeting ZFAS1-mRNA pairs. The Pearson correlation coefficient was used as the threshold for selecting co-expressed ZFAS1-gene pairs. An absolute value of the Pearson correlation coefficient of $\geq 0.3$ was used as the threshold. The Search Tool for the Retrieval of Interacting Genes/Proteins version 10.0 (STRING; string-db.org) $(12,13)$ was used for the exploration of potential ZFAS1 protein interactions. The PPI networks of DEGs generated by STRING were derived from validated experiments. A combined score of $>0.9$ was considered significant. $\mathrm{P}<0.05$ was considered to indicate a statistically significant difference. The PPI networks were visualized using Cytoscape (14).

Gene Ontology (GO) and Kyoto Encyclopedia of Genes and Genomes (KEGG) pathway analysis. MAS 3.0 (mas. capitalbiotech.com/mas3) may be used to analyze inter-gene correlations and for gene function annotation. In the present study, the DAVID tool (https://david.ncifcrf.gov/tools.jsp) was used to perform GO and KEGG pathway analysis using the genes co-expressed with ZFAS1. $\mathrm{P}<0.05$ was selected as the threshold of significance.

Cell culture. The OVCA429, SKOV3, A2780 and COV644 OC cell lines and normal human ovarian surface epithelial (HOSE) cells were purchased from the Cell Bank of the Chinese Academy of Sciences. All cell lines were verified by using short tandem repeat analysis. The OVCA429, SKOV3, A2780 and COV644 cell lines were cultured in Dulbecco's modified Eagle's medium (Gibco-BRL; Thermo Fisher Scientific, Inc.) supplemented with $10 \%$ fetal bovine serum (Gibco; Thermo Fisher Scientific, Inc.) and antibiotics (Gibco-BRL; Thermo Fisher Scientific, Inc.). HOSE cells were cultured in medium composed of a 1:1 mixture of MCDB 105 (Sigma-Aldrich, Merck KGaA) and M199 (Gibco;
Thermo Fisher Scientific, Inc.) medium containing 10\% fetal bovine serum (Gibco; Thermo Fisher Scientific, Inc.). Cells were cultured in a humidified atmosphere containing $5 \% \mathrm{CO}_{2}$ at $37^{\circ} \mathrm{C}$.

Transfection. Lipofectamine 3000 (Invitrogen; Thermo Fisher Scientific, Inc.) was used to perform transfection according to the manufacturer's instructions. The SKOV3 cells were transfected with siZFAS1 and siNC at a final concentration of $50 \mathrm{nM}$. After $48 \mathrm{~h}$, the cells were used to perform cell counting kit-8 (CCK-8) and cell cycle assays. The following siRNA sequences were used: siZFAS1, 5'-AGCGGTTTGGTGCGG TGTGAAGCGCGACAT-3' and siNC, 5'-UUCUCCGAACGU GUCACGUdTdT-3'.

CCK- 8 viability assay. The transfected cells $\left(3 \times 10^{3}\right.$ cells/well $)$ were seeded in 96-well culture plates and incubated with $10 \mu \mathrm{l}$ CCK-8 reagent (Beyotime Institute of Biotechnology) per well at $37^{\circ} \mathrm{C}$ for $2 \mathrm{~h}$. Viability ability was detected at the selected time points ( $0,1,2$ and 3 days following seeding). The optical density was determined at a wavelength of $450 \mathrm{~nm}$.

Reverse transcription-quantitative $(R T-q) P C R$ analysis. For RT-qPCR, an Ultrapure RNA kit (CWBIO) was used to extract RNA. A PrimeScript RT reagent kit (Takara) was used to perform RT. Reaction parameters were as follows: $42^{\circ} \mathrm{C}$ for $15 \mathrm{~min}, 85^{\circ} \mathrm{C}$ for $5 \mathrm{sec}$ and $4^{\circ} \mathrm{C}$ for $5 \mathrm{~min}$. AceQ Universal SYBR qPCR Master Mix (Vazyme) was used to perform Real-time PCR on an ABI 7500 system (Applied Biosystems; Thermo Fisher Scientific, Inc.). Reaction parameters were as follows: Initial denaturation at $95^{\circ} \mathrm{C}$ for $5 \mathrm{~min}$; 40 cycles of $95^{\circ} \mathrm{C}$ for $10 \mathrm{sec}$ and $60^{\circ} \mathrm{C}$ for $30 \mathrm{sec}$. The sequences of the primers were as follows: ZFAS1 forward, 5'-ACGTGCAGA CATCTACAACCT-3' and reverse, 5'-TACTTCCAACACCCG CAT-3'; $\beta$-actin forward, 5'-GAGCTACGAGCTGCCTGA CG-3' and reverse, 5'-CCTAGAAGCATTTGCGGTGG-3'. $\beta$-actin was used as a reference. The $2^{-\Delta \Delta \mathrm{Cq}}$ method was used to quantify the data (15).

Statistical analysis. Statistical analysis was performed using SPSS 19.0 software (IBM Corp.). Student's t-test or Mann-Whitney U-test was used to perform statistical comparisons between two groups depending on the test condition. One-way analysis of variance was used to perform statistical comparisons among multiple groups followed by Dunnett's post-hoc test. $\mathrm{P}<0.05$ was considered to indicate statistical significance.

\section{Results}

LncRNA ZFAS1 is upregulated in advanced-vs. early-stage $O C$. In order to assess the roles of ZFAS1 in OC, the expression levels of ZFAS1 in OC and normal samples were first analyzed using the GEPIA dataset. Of note, the analysis revealed that ZFAS1 was significantly downregulated in OC compared with that in normal samples (Fig. 1A). This result was not consistent with that of a previous study (7).

It was then assessed whether ZFAS1 expression is associated with the stage of OC by analyzing TCGA RNA-seq dataset. As presented in Fig. 1B, ZFAS1 was overexpressed 


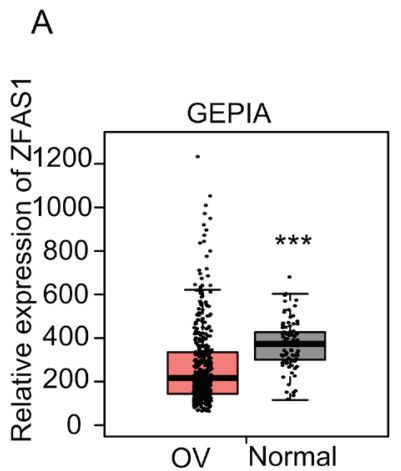

D

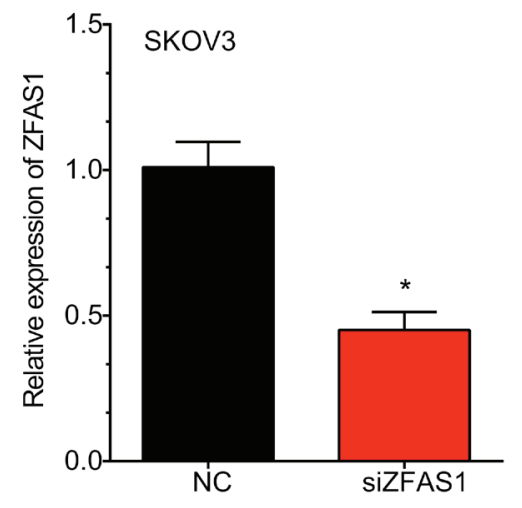

F

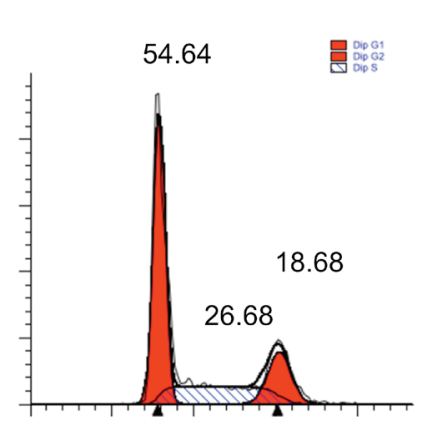

B

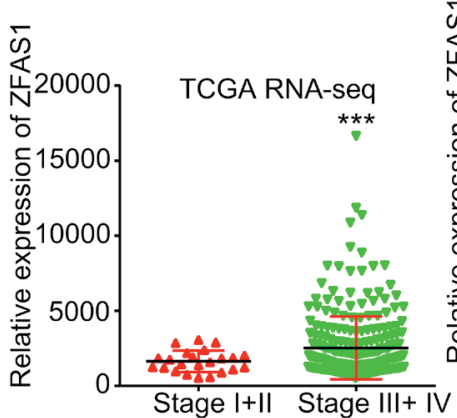

E

C

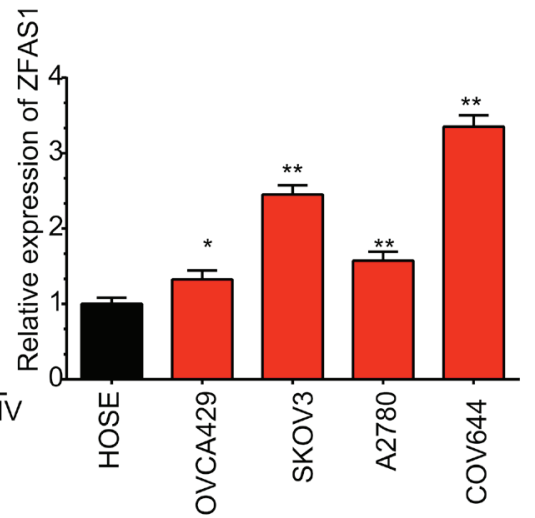

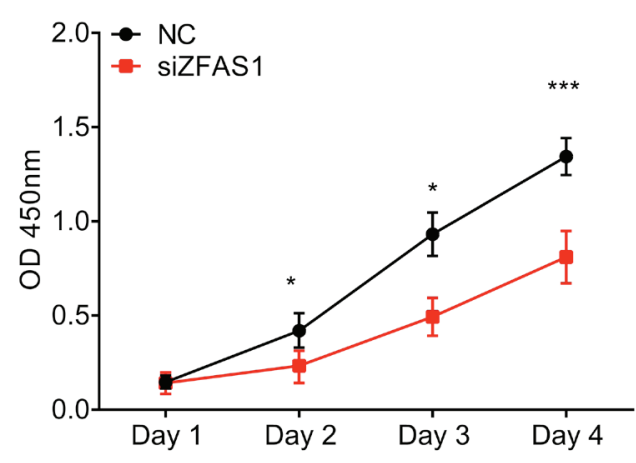

G

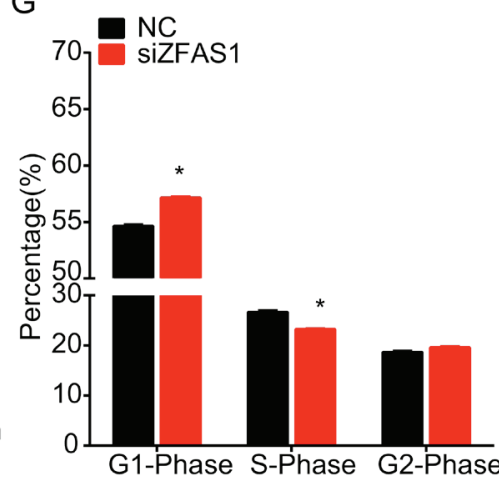

Figure 1. ZFAS1 is upregulated in OC vs. normal ovarian cells and in advanced- vs. early-stage OC tissues. (A) In the GEPIA dataset, ZFAS1 was downregulated in OC compared to normal samples. ${ }^{* * *} \mathrm{P}<0.05$ vs. OC. (B) In TCGA RNA-seq dataset, ZFAS1 was overexpressed at higher-stage (stage III/IV) OC compared with that in the early-stage OC (stage I/II) samples. ${ }^{* * *} \mathrm{P}<0.001$ vs. stage I+II. (C) ZFAS1 was significantly upregulated in the OVCA429, SKOV3, A2780 and COV644 OC cell lines compared with that in normal HOSE cells. "P $<0.05$ and ${ }^{* *} \mathrm{P}<0.01$ vs. HOSE. (D) Silencing efficiency. (E) Knockdown of ZFAS1 markedly inhibited SKOV3 cell proliferation. (F) Knockdown of ZFAS1 in SKOV3 cells significantly increased the percentage of cells in G1 phase, but decreased the percentage of cells in S phase. (G) Cell cycle data were expressed as the mean \pm standard deviation. ${ }^{*} \mathrm{P}<0.05$ and ${ }^{* * * *} \mathrm{P}<0.001 \mathrm{vs}$. NC. OC, ovarian cancer; ZFAS1, zinc finger nuclear transcription factor, X-box binding 1-type containing 1 antisense RNA 1; HOSE, human ovarian surface epithelium; TCGA, The Cancer Genome Atlas; NC, negative control; siZFAS1, small interfering RNA targeting ZFAS1; OD, optical density; RNA-seq, RNA sequencing; GEPIA, Gene Expression Profiling Interactive Analysis.

at higher stages (stage III/IV) of OC compared with that in early-stage (stage I/II) OC samples.

The expression of ZFAS1 in OC cell lines was detected by RT-qPCR. It was revealed that the expression levels of ZFAS1 were significantly higher in the OVCA429, SKOV3, A2780 and COV644 cell lines than those in normal HOSE cells (Fig. 1C).

Knockdown of ZFAS1 suppresses SKOV3 cell viability. The effect of ZFAS1 on OC cell viability was detected using a Cell Counting Kit (CCK)-8 assay following ZFAS1 knockdown. The silencing efficiency was confirmed using RT-qPCR analysis (Fig. 1D). As presented in Fig. 1E, knockdown of ZFAS1 markedly inhibited SKOV 3 cell viability at $72 \mathrm{~h}$ post-transfection. Cell cycle analysis demonstrated that knockdown of ZFAS1 in SKOV3 cells significantly increased the percentage of cells in G1 phase, but decreased the percentage of cells in $\mathrm{S}$ phase (Fig. 1F and G). These results suggested that knockdown of ZFAS1 suppressed SKOV3 cell viability by inducing cell cycle arrest.

Higher ZFAS1 expression in OC tissues is associated with poor prognosis. The association between ZFAS1 expression 
A

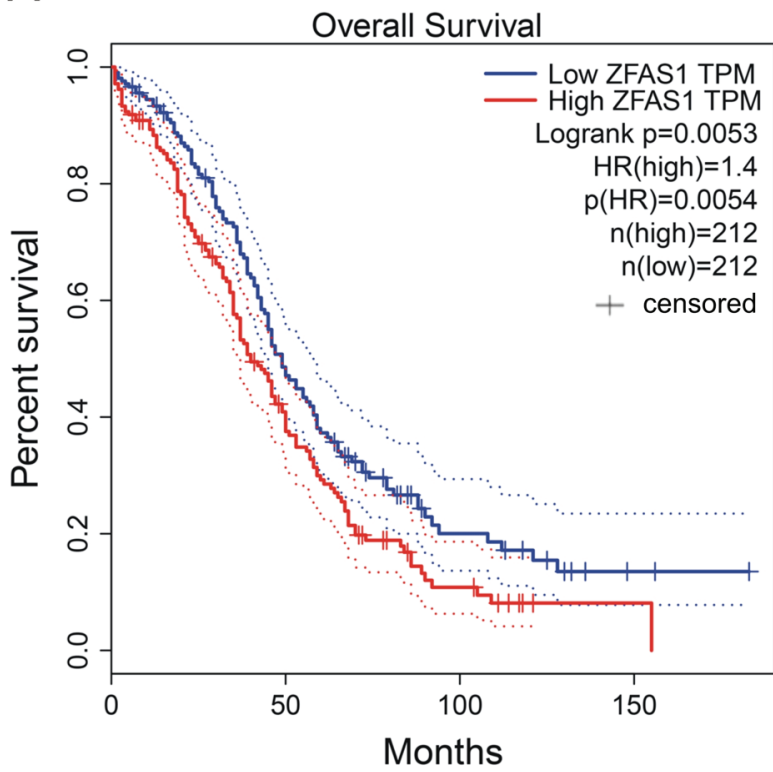

C

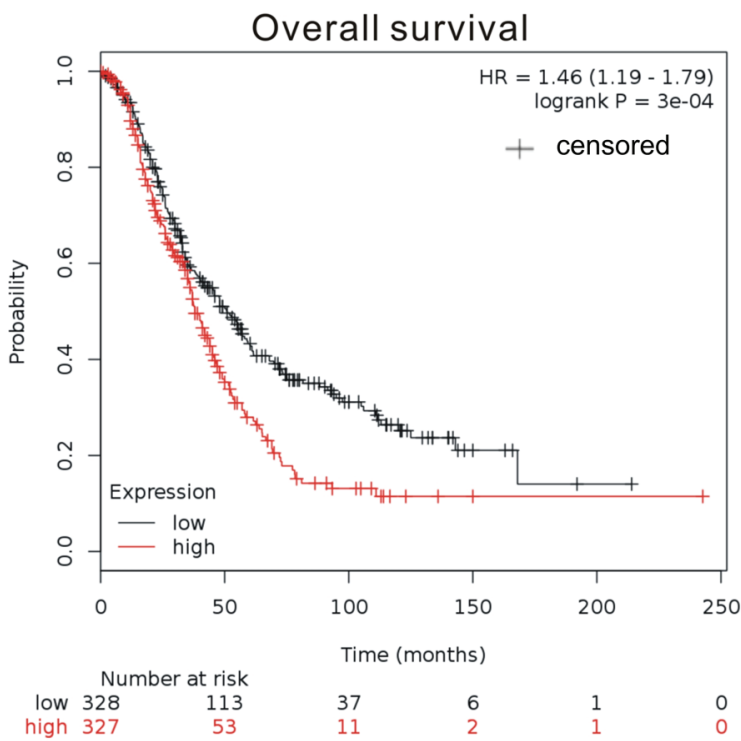

B

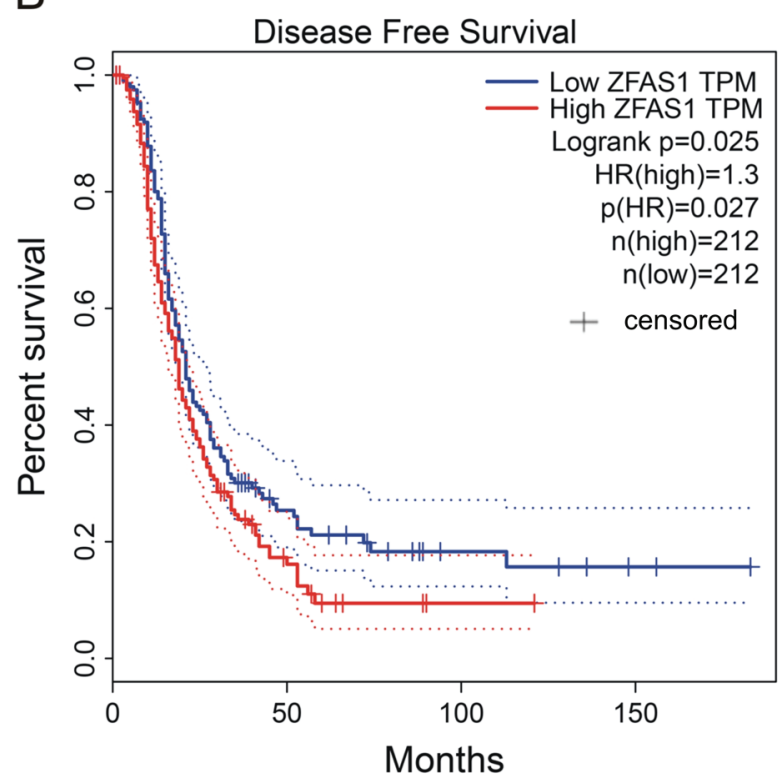

D

Kaplan Meier plotter

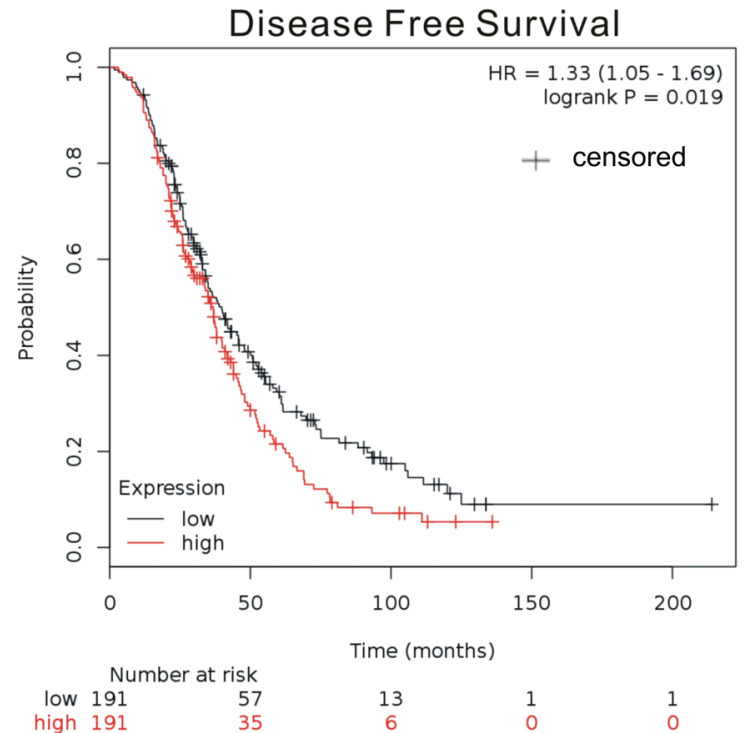

Figure 2. Higher ZFAS1 expression is associated with poor prognosis in ovarian cancer. (A and B) TCGA dataset and (C and D) Kaplan-Meier plotter analysis indicated that higher ZFAS1 expression was associated with shorter (A and C) overall survival time and (B and D) disease-free survival time. The expression of ZFAS1 in OC tissues was categorized as high or low according to the median value. ZFAS1, zinc finger nuclear transcription factor, X-box binding 1-type containing 1 antisense RNA 1; TCGA, The Cancer Genome Atlas; HR, hazard ratio; TPM, transcripts per kilobase million.

and prognosis was investigated using TCGA and Kaplan-Meier plotter datasets. The expression of ZFAS1 in OC tissues was categorized as high or low according to the median value. By analyzing TCGA datasets, it was revealed that higher ZFAS1 expression levels were associated with shorter overall survival time (OS; Fig. 2A) and disease-free survival time (DFS; Fig. 2B).

Next, the Kaplan-Meier plotter datasets, which included 1,287 OC samples with a mean OS of 31 months were analyzed. In this dataset, OC patients with higher ZFAS1 expression had a shorter OS and DFS when compared with patients with low expression (Fig. 2C and D). These results suggested that ZFAS1 may serve as a novel prognostic marker in OC patients.
Construction of ZFAS1-associated PPI networks. In the present study, a PPI network of ZFAS1 was first generated by calculating the Pearson correlation coefficient between ZFAS1 and mRNAs in TCGA OC datasets. ZFAS1-mRNA pairs with an absolute value of the Pearson correlation coefficient of $\geq 0.3$ were selected.

Next, ZFAS1-mediated PPI networks in OC were constructed by using the STRING database (combined score $>0.9$ ). A total of 628 proteins and 12,158 edges were included in the PPI network of genes positively co-expressed with ZFAS1. Of note, several hub genes were identified in this network, as presented in Fig. 3. Module 1 contained 104 proteins and 5,096 edges (Fig. 3A), Module 2 contained 47 proteins and 1,018 edges (Fig. 3B), Module 3 contained 39 proteins and 732 edges (Fig. 3C), Module 4 contained 36 proteins and 602 edges (Fig. 3D) and 


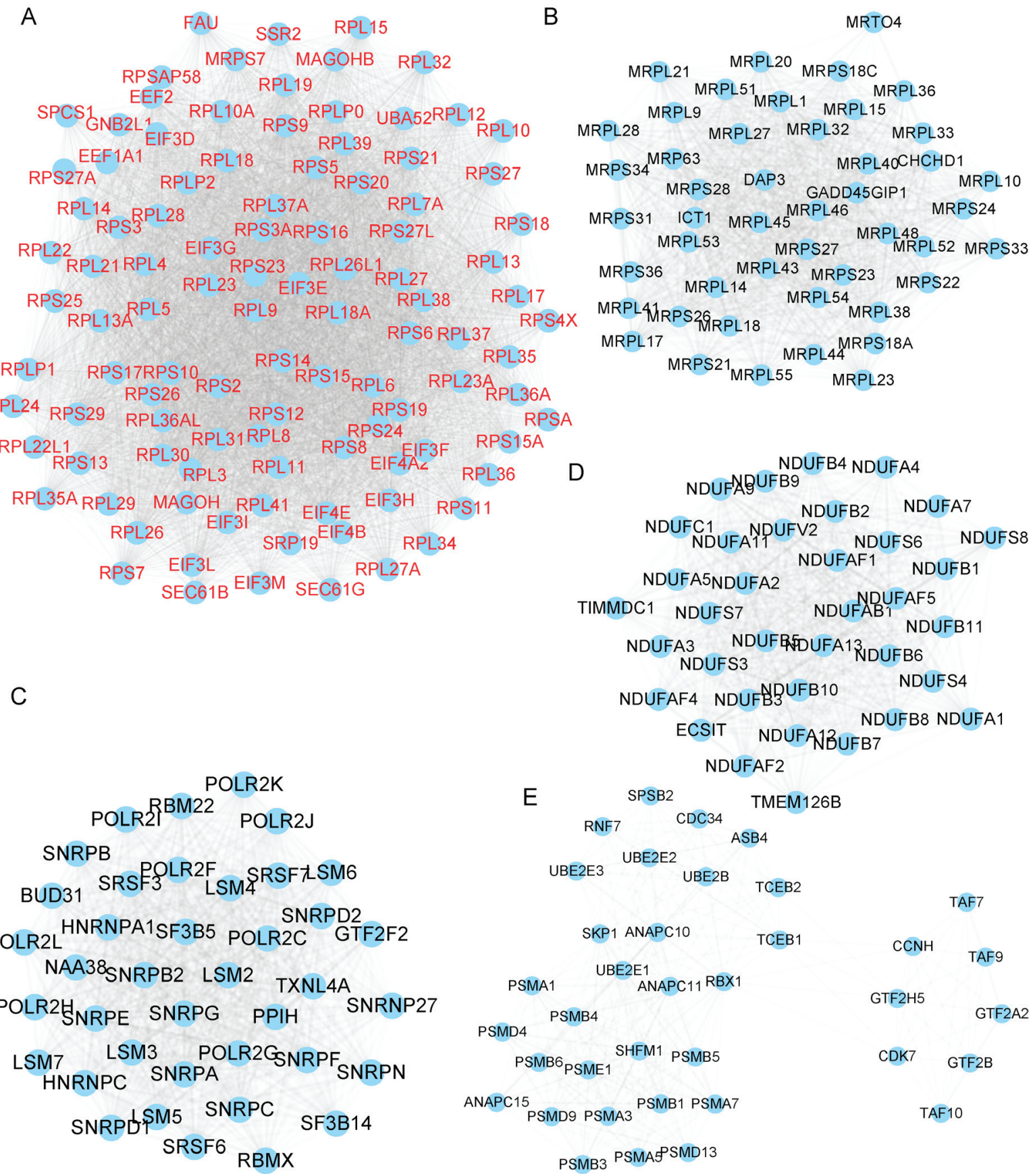

Figure 3. Protein-protein interaction network of proteins positively co-expressed with zinc finger nuclear transcription factor, X-box binding 1-type containing 1 antisense RNA 1. (A) Module 1 contained 104 proteins and 5,096 edges, (B) Module 2 contained 47 proteins and 1,018 edges, (C) Module 3 contained 39 proteins and 732 edges, (D) Module 4 contained 36 proteins and 602 edges and (E) Module 5 contained 37 proteins and 298 edges.

Module 5 contained 37 proteins and 298 edges (Fig. 3E). A total of 277 proteins and 1,216 edges were included in the PPI network of genes negatively co-expressed with ZFAS1 (Fig. 4).

Construction of ZFAS1-mediated competing endogenous (ce)RNA network in OC. Previous studies have indicated that ZFAS1 acts as a ceRNA in human cancers. In order to explore the potential mechanism by which ZFAS1 regulates OC progression, a ZFAS1-mediated ceRNA network was constructed. The Starbase and TargetScan databases were used to predict the miRNAs targeting ZFAS1-mRNA pairs.

As presented in Fig. 5A, this ceRNA network included 2 miRNAs [Homo sapiens (hsa)-miR-150-5p and
hsa-miR-590-3p] and 195 mRNAs. hsa-miR-150-5p has been previously reported to be a target of ZFAS1 in OC (7). The correlation between ZFAS1 and the candidate miRNAs was then analyzed. It was revealed that ZFAS1 was negatively correlated to the expression of hsa-miR-150-5p and hsa-miR-590-3p in OC (Fig. 5B and C). However, the expression of ZFAS1 was not significantly correlated to miR-329 expression in OC (Fig. 5D), which was previously reported to be sponged by ZFAS1 in bladder cancer (16).

Enrichment analysis of ZFAS1 in OC. Enrichment analysis of genes co-expressed with ZFAS1 was then performed by using the DAVID tool. GO analysis indicated that genes positively 


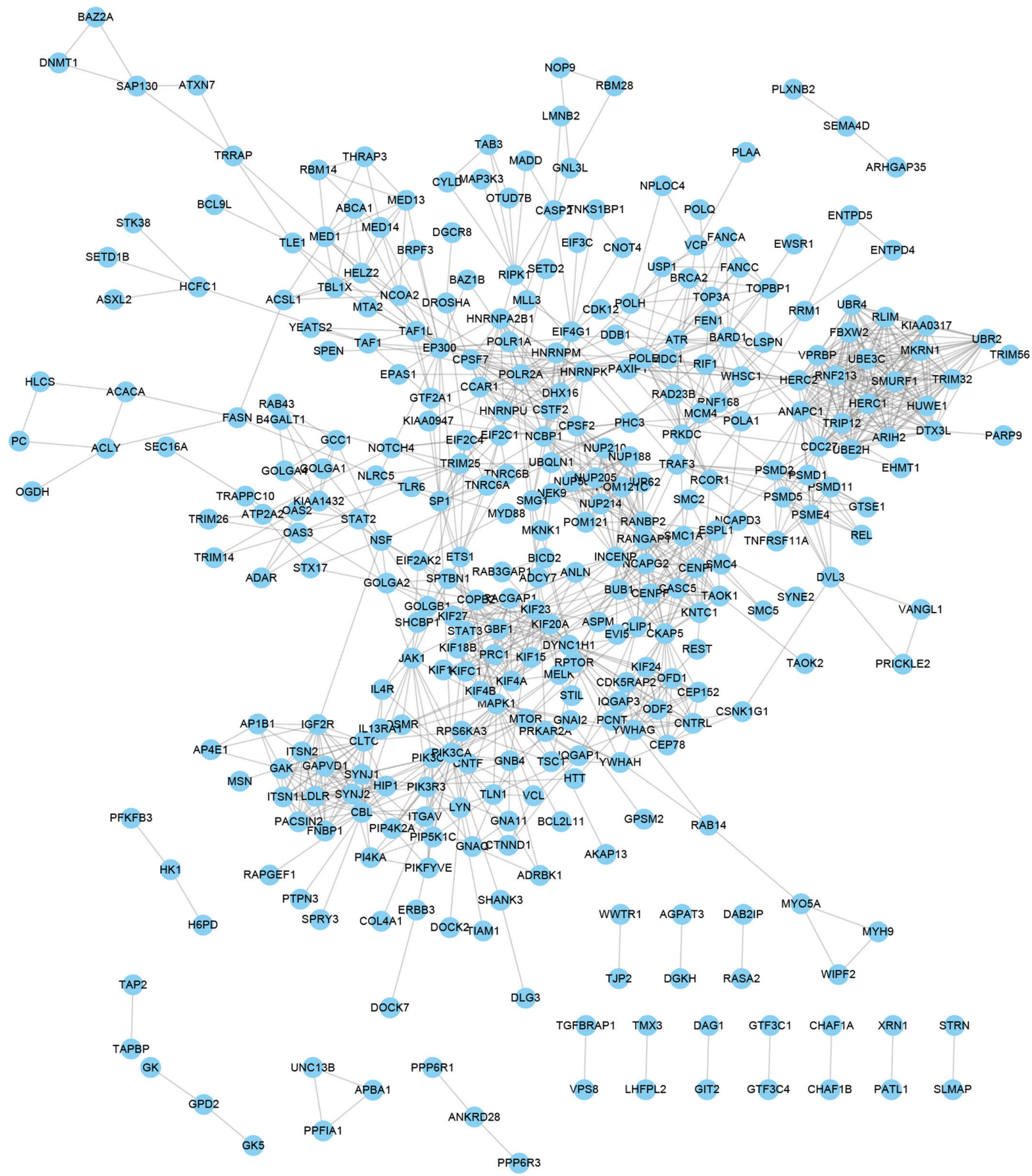

Figure 4. Protein-protein interaction network of proteins negatively co-expressed with zinc finger nuclear transcription factor, X-box binding 1-type containing 1 antisense RNA 1. A total of 277 proteins and 1,216 edges were included. ZFAS1, zinc finger nuclear transcription factor, X-box binding 1-type containing 1 antisense RNA 1; PPI, protein-protein interaction.

co-expressed with ZFAS1 are associated with translation, translational initiation, ribosomal RNA processing, mRNA splicing and hydrogen ion transmembrane transport (Fig. 6A). KEGG analysis suggested that genes positively co-expressed with ZFAS1 are associated with the pathways of ribosome, oxidative phosphorylation, spliceosome, RNA transport and RNA polymerase (Fig. 6B).

GO analysis revealed that genes negatively co-expressed with ZFAS1 were associated with cell-cell adhesion, DNA repair, protein sumoylation, positive regulation of GTPase activity and DNA replication (Fig. 6C). KEGG analysis indicated that genes negatively co-expressed with ZFAS1 were associated with the insulin, thyroid hormone, AMP kinase, mTOR and hypoxia-inducible factor-1 signaling pathways (Fig. 6D).

\section{Discussion}

OC is one of the most fatal types of gynecological malignancy. Of note, the prognosis of OC has remained poor with the 5-year 
A

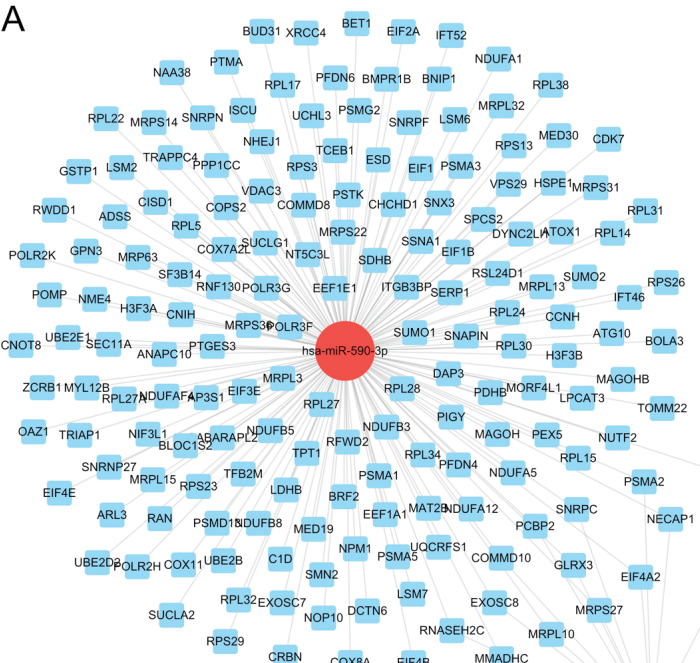

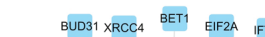

RPL22 MPPS14 SNRPN 1 ISOU

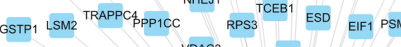

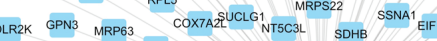

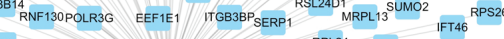

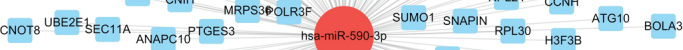

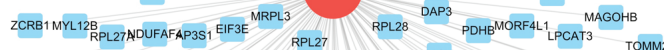

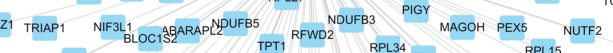

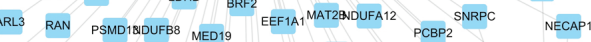

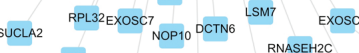

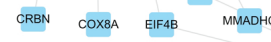

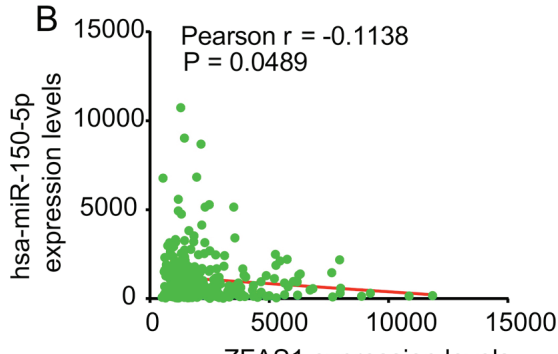

C

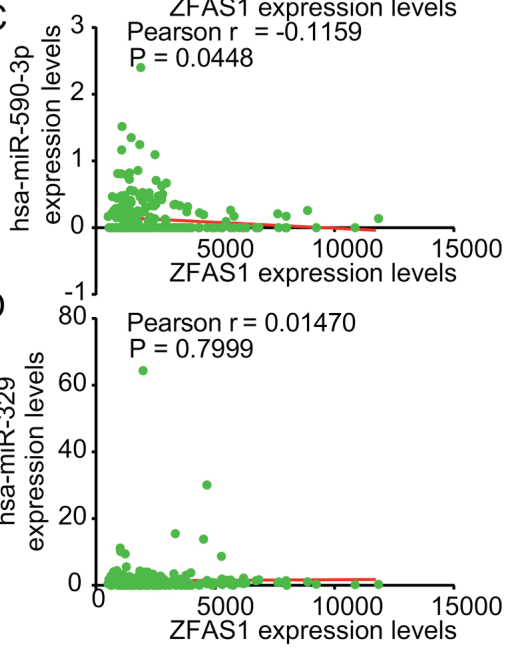

Figure 5. ZFAS1-mediated ceRNA network in ovarian cancer. (A) ZFAS1-mediated ceRNA network. Correlation between ZFAS1 and candidate miRNAs (B) hsa-miR-150-5p, (C) hsa-miR-590-3p and (D) miR-329. ZFAS1, zinc finger nuclear transcription factor, X-box binding 1-type containing 1 antisense RNA 1; miR, microRNA; hsa, Homo sapiens; ceRNA, competing endogenous RNA.

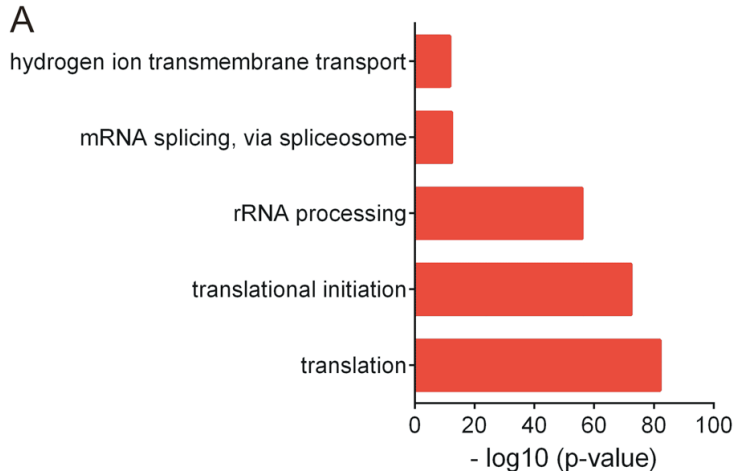

C

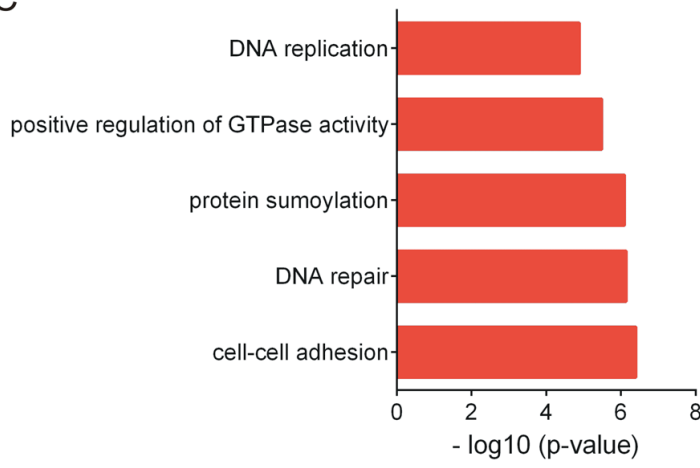

B

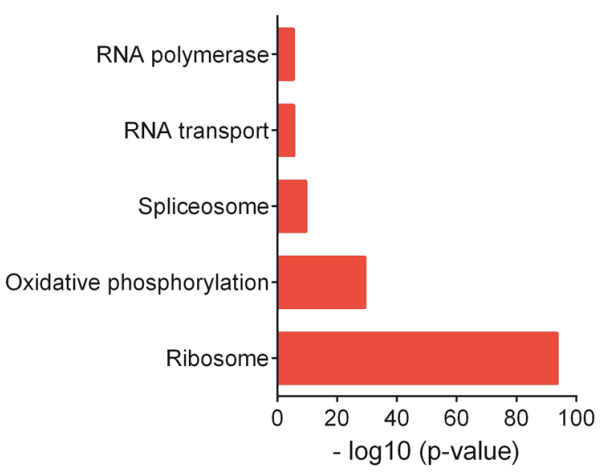

$\mathrm{D}$

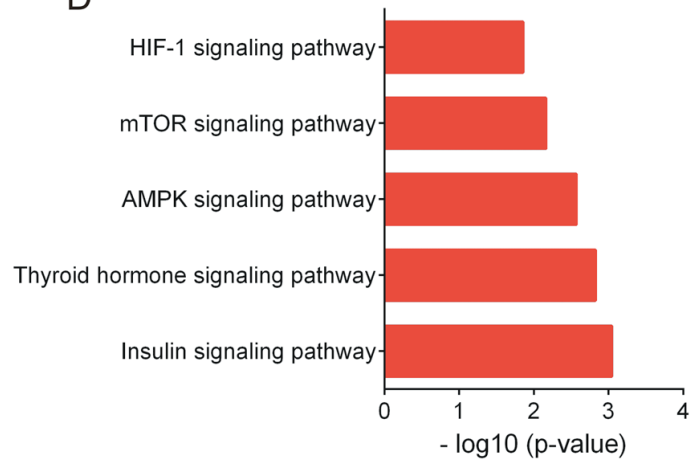

Figure 6. Functional analysis of genes co-expressed with ZFAS1. (A) GO analysis of positively correlated co-expressed genes. (B) KEGG analysis of positively correlated co-expressed genes. (C) GO analysis of negatively correlated co-expressed genes. (D) KEGG analysis negatively correlated co-expressed genes.

survival rate of advanced-stage OC being $<30 \%$. LncRNAs act as novel regulators in cancer progression and were observed to be differentially expressed in various human cancer types, including OC. Certain lncRNAs have been revealed to have 
crucial roles in OC by regulating cell viability, metastasis and resistance to chemotherapeutics. For instance, lncRNA small NF90-associated RNA was reported to promote OC viability by upregulating growth factor receptor-bound protein 2-associated binding protein (17), IncRNA prostate cancer gene expression marker 1-induced $\mathrm{OC}$ tumorigenesis through the Ras homolog family member pathway (18) and metastasis-associated lung adenocarcinoma transcript 1 regulated OC cell viability, migration and apoptosis through the phosphoinositide 3-kinase/AKT pathway (19). Of note, the expression pattern and functional roles of the vast majority of IncRNAs in OC have remained elusive.

ZFAS1 has been reported to be a potential biomarker in HCC, colorectal cancer, gastric cancer and glioma. For instance, Wang and Xing (20) reported that upregulation of ZFAS1 in colorectal cancer tissues predicted poor prognosis. Furthermore, a meta-analysis by Song et al (21) indicated that high ZFAS1 expression in solid tumors was associated with a shorter OS and recurrence-free survival. However, the expression pattern of ZFAS1 in OC has remained largely elusive. In the present study, the expression levels of ZFAS1 in OC and normal samples was first analyzed using the GEPIA dataset. It was revealed that ZFAS1 was downregulated in OC compared to normal samples. This result was not consistent with that of a previous study by Xia et al (7), reporting that ZFAS1 was upregulated in OC (7). Thus, the present study hypothesized that ZFAS1 expression may be associated with the progression of OC. The results of TCGA analysis suggested that ZFAS1 was overexpressed in advanced-stage OC compared with that in early-stage OC samples. Furthermore, by detecting ZFAS1 expression levels in OC cell lines, it was revealed that ZFAS1 was overexpressed in OC cell lines. GEPIA was based on TCGA and Genotype-Tissue Expression project (GTEx) data. The TCGA and GTEx data were produced by two different groups, which may be a possible reason why the results of the GEPIA analysis were not consistent with those of Xia et al (7). In order to validate whether ZFAS1 may serve as a prognostic marker for OC, the association between ZFAS1 expression in OC tissues and survival time was then analyzed, revealing that OC patients with higher ZFAS1 expression had a shorter OS and DFS. Taken together, these results suggested that ZFAS1 may serve as a novel biomarker for OC.

LncRNAs exert their roles in cancer cells by interacting with other RNA molecules, proteins and DNA. ZFAS1 was reported to promote the occurrence of nasopharyngeal carcinoma by activating the $\mathrm{Wnt} / \beta$-catenin pathway (22). Knockdown of ZFAS1 repressed cell viability via inducing KLF2 and NKD2 expression and inhibited cell migration and invasion via reducing ZEB1 and ZEB2 expression in gastric cancer (5). ZFAS1 was also identified to be a ceRNA by sponging miR-329 in bladder cancer (9), miR-940 in prostate cancer (23), miR-486 in osteosarcoma (24) and miR-484 in colorectal cancer (25). In the present study, a loss-of-function assay was performed to examine the effect of ZFAS1 on OC viability. The results suggested that knockdown of ZFAS1 significantly suppressed OC cell viability and induced cell cycle arrest. These in vitro results were consistent with previous study and showed that ZFAS1 played as an oncogene in OC. In addition, a Bioinformatics analysis was performed to reveal the potential mechanisms underlying the role of ZFAS1 in OC progression. The proteins co-expressed with ZFAS1 were determined and PPI networks were constructed from them. Furthermore, a Bioinformatics analysis was performed to determine the GO terms and KEGG pathways enriched by genes co-expressed with ZFAS1. GO analysis indicated that the genes positively co-expressed with ZFAS1 are associated with translation, mRNA splicing, cell-cell adhesion, DNA repair, protein sumoylation, positive regulation of GTPase activity and DNA replication. In addition, a ZFAS1-mediated ceRNA network was constructed in OC, which included 2 miRNAs (hsa-miR-150-5p and hsa-miR-590-3p) and 195 mRNAs. Of note, Xia et al (7) also reported that ZFAS1 interacted with miR-150-5p to promote $\mathrm{Sp} 1$ expression. The present analyses were consistent with the study by Xia et al (7) in terms of ZFAS1 being upregulated in OC and miR-150-5p being a target of ZFAS1. The present study provided novel information contributing to the understanding of the functions of ZFAS1. For instance, it indicated that miR-590-3p was also a potential target of ZFAS1, which may regulate $>150$ ZFAS1 co-expressing genes in OC.

In conclusion, the present study indicated that ZFAS1 was overexpressed in OC compared with normal cells. By analyzing GEPIA dataset, the present study found that ZFAS1 was downregulated in OC compared with normal samples; however, it was highly expressed in advanced stage OC compared to early stage OC samples. Higher ZFAS1 expression was associated with shorter OS and DFS in OC patients. Knockdown of ZFAS1 suppressed SKOV3 cell viability and cell cycle progression. Bioinformatics analysis indicated that ZFAS1 is associated with translation, mRNA splicing, cell-cell adhesion, DNA repair, protein sumoylation, positive regulation of GTPase activity and DNA replication. The present study suggests that ZFAS1 may serve as a biomarker for OC.

\section{Acknowledgements}

Not applicable.

\section{Funding}

No funding was received.

\section{Availability of data and materials}

The datasets analyzed during the current study are available in the Genomic Data Commons Data Portal repository [https://portal.gdc.cancer.gov/exploration?filters $=\% 7 \mathrm{~B} \% 22 \mathrm{op} \%$ $22 \% 3 \mathrm{~A} \% 22$ and $\% 22 \% 2 \mathrm{C} \% 22$ content $\% 22 \% 3 \mathrm{~A} \% 5 \mathrm{~B} \% 7 \mathrm{~B} \% 22 \mathrm{op}$ $\% 22 \% 3 \mathrm{~A} \% 22$ in $\% 22 \% 2 \mathrm{C} \% 22$ content $\% 22 \% 3 \mathrm{~A} \% 7 \mathrm{~B} \% 22$ field $\% 2$ $2 \% 3 \mathrm{~A} \% 22$ cases.primary_site $\% 22 \% 2 \mathrm{C} \% 22$ value $\% 22 \% 3 \mathrm{~A} \% 5 \mathrm{~B}$ \%22Ovary\%22\%5D\%7D\%7D\%5D\%7D].

\section{Authors' contributions}

SH and MFX conceived and designed the study. SH, DZL and MFX developed the methodology, analysed and interpreted the data, and wrote, reviewed and/or revised the manuscript. 


\section{Ethics approval and consent to participate}

Not applicable.

\section{Patient consent for publication}

Not applicable.

\section{Competing interests}

The authors declare that they have no competing interests.

\section{References}

1. Siegel RL, Miller KD and Jemal A: Cancer statistics, 2017. CA Cancer J Clin 671: 7-30, 2017.

2. Yang G, Lu X and Yuan L: LncRNA: A link between RNA and cancer. Biochim Biophys Acta 1839: 1097-1109, 2014.

3. Ma J and Xue M: LINK-A IncRNA promotes migration and invasion of ovarian carcinoma cells by activating TGF- $\beta$ pathway. Biosci Rep 38: pii: BSR20180936, 2018.

4. Shu C, Yan D, Mo Y, Gu J, Shah N and He J: Long noncoding RNA lncARSR promotes epithelial ovarian cancer cell proliferation and invasion by association with HuR and miR-200 family. Am J Cancer Res 86: 981-992, 2018.

5. Nie F, Yu X, Huang M, Wang Y, Xie M, Ma H, Wang Z, De W and Sun M: Long noncoding RNA ZFAS1 promotes gastric cancer cells proliferation by epigenetically repressing KLF2 and NKD2 expression. Oncotarget 824: 38227-38238, 2017.

6. Fang C, Zan J, Yue B, Liu C, He C and Yan D: Long non-coding ribonucleic acid zinc finger antisense 1 promotes the progression of colonic cancer by modulating ZEB1 expression. J Gastroenterol Hepatol 326: 1204-1211, 2017.

7. Xia B, Hou Y, Chen H, Yang S, Liu T, Lin M and Lou G: Long non-coding RNA ZFAS1 interacts with miR-150-5p to regulate Sp1 expression and ovarian cancer cell malignancy. Oncotarget 8: 19534-19546, 2017.

8. Tang Z, Li C, Kang B, Gao G, Li C and Zhang Z: GEPIA: A web server for cancer and normal gene expression profiling and interactive analyses. Nucleic Acids Res 45: W98-W102, 2017.

9. Li JH, Liu S, Zhou H, Qu LH and Yang JH: starBase v2.0: Decoding miRNA-ceRNA, miRNA-ncRNA and protein-RNA interaction networks from large-scale CLIP-Seq data. Nucleic Acids Res 42 (Database Issue): D92-D97, 2014.

10. Agarwal V, Bell GW, Nam JW and Bartel DP: Predicting effective microRNA target sites in mammalian mRNAs. eLife 4: e05005, 2015.

11. Garcia DM, Baek D, Shin C, Bell GW, Grimson A and Bartel DP Weak seed-pairing stability and high target-site abundance decrease the proficiency of lsy- 6 and other microRNAs. Nat Struct Mol Biol 18: 1139-1146, 2011.
12. Szklarczyk D, Morris JH, Cook H, Kuhn M, Wyder S, Simonovic M, Santos A, Doncheva NT, Roth A, Bork P, et al: The STRING database in 2017: Quality-controlled protein-protein association networks, made broadly accessible. Nucleic Acids Res 45: D362-D368, 2017.

13. Franceschini A, Lin J, von Mering C and Jensen LJ: SVD-phy: Improved prediction of protein functional associations through singular value decomposition of phylogenetic profiles. Bioinformatics 32: 1085-1087, 2016.

14. Shannon P, Markiel A, Ozier O, Baliga NS, Wang JT, Ramage D, Amin N, Schwikowski B and Ideker T: Cytoscape: A software environment for integrated models of biomolecular interaction networks. Genome Res 13: 2498-2504, 2003.

15. Livak KJ and Schmittgen TD: Analysis of relative gene expression data using real-time quantitative PCR and the 2(-Delta Delta C(T)) method. Methods 254: 402-408, 2001.

16. Wang JS, Liu QH, Cheng XH, Zhang WY and Jin YC: The long noncoding RNA ZFAS1 facilitates bladder cancer tumorigenesis by sponging miR-329. Bio Pharm 103: 174-181, 2018.

17. Huang $\mathrm{Y}, \mathrm{Hu} \mathrm{Y}$, Jin $\mathrm{Z}$ and Shen Z: LncRNA snaR upregulates GRB2-associated binding protein 2 and promotes proliferation of ovarian carcinoma cells. Biochem Biophys Res Commun 503: 2028-2032, 2018

18. Chen S, Wang LL, Sun KX, Liu Y, Guan X, Zong ZH and Zhao Y: LncRNA PCGEM1 induces ovarian carcinoma tumorigenesis and progression through RhoA pathway. Cell Physiol Biochem 474: 1578-1588, 2018.

19. Jin Y, Feng SJ, Qiu S, Shao N and Zheng JH: LncRNA MALAT1 promotes proliferation and metastasis in epithelial ovarian cancer via the PI3K-AKT pathway. Eur Rev Med Pharmacol Sci 2114: 3176-3184, 2017

20. Wang W and Xing C: Upregulation of long noncoding RNA ZFAS1 predicts poor prognosis and prompts invasion and metastasis in colorectal cancer. Pathol Res Pract 2128: 690-695, 2016.

21. Song W, Tian C, Zhang RJ, Zou SB and Wang K: Meta-analysis of the prognostic value of lncRNA ZFAS1 in patients with solid tumors. Oncotarget 852: 90301-90307, 2017.

22. Chen X, Li J, Li CL and Lu X: Long non-coding RAN ZFAS promotes nasopharyngeal carcinoma through activation of Wnt $/ \beta$-catenin pathway. Eur Rev Med Pharmacol Sci 2211: 3423-3429, 2018

23. Chen X, Yang C, Xie S and Cheung E: Long non-coding RNA GAS5 and ZFAS1 are prognostic markers involved in translation targeted by miR-940 in prostate cancer. Oncotarget 91: 1048-1062, 2018

24. Li N, Sun ZH, Fang M, Xin JY and Wan CY: Long non-coding RNA ZFAS1 sponges miR-486 to promote osteosarcoma cells progression and metastasis in vitro and vivo. Oncotarget 861: 104160-104170, 2017.

25. Xie S, Ge Q, Wang X, Sun X and Kang Y: Long non-coding RNA ZFAS1 sponges miR-484 to promote cell proliferation and invasion in colorectal cancer. Cell Cycle 172: 154-161, 2018.

(i) $\ominus$ This work is licensed under a Creative Commons Attribution-NonCommercial-NoDerivatives 4.0 International (CC BY-NC-ND 4.0) License. 